\title{
Initial Operation and Beam Characteristics of the UCLA S-band RF Photo-Injector*
}

\author{
C. Pellegrini, N. Barov, S. C. Hartman, S. Park,
}

J. Rosenzweig, G. Travish, R. Zhang

Department of Physics, University of California, Los Angeles 90024

P. Davis, C. Joshi, G. Hairapetian

Electrical Engineering Department, University of California, Los Angeles 90024

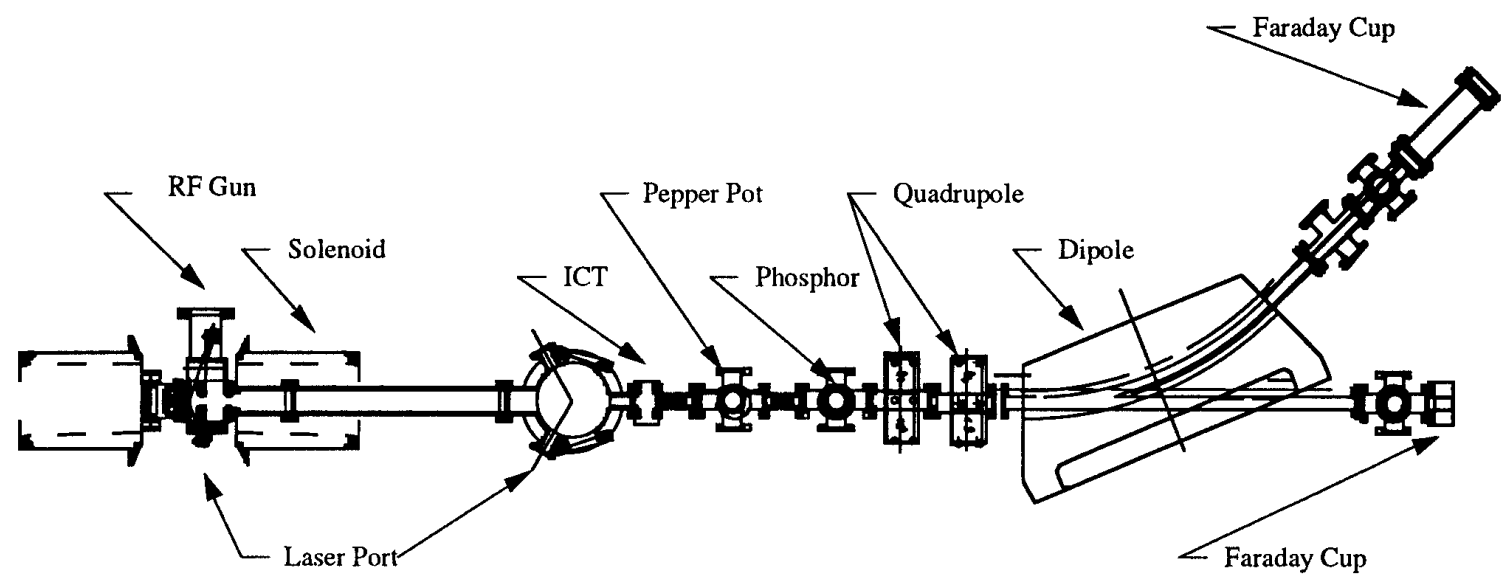

Figure 1.

\section{Abstract}

The UCLA rf photo-injector system has been commissioned(1). All of the sub-components such as the high power rf, pico-second laser, rf photo-injector cavity, diagnostics, and supporting hardware have been tested and are operational. We briefly discuss the performance of the various components since the details of each subsystem are very lengthy. The laser delivers a sub 4 ps pulse containing 0 $300 \mu \mathrm{J}$ of energy per pulse. The photo-injector produces $0-3$ $\mathrm{nC}$ per bunch with an $\mathrm{rf}$ induced emittance of $1.5 \pi(\mathrm{mm}$ mrad).

\section{INTRODUCTION}

We report the initial results of the operation of the UCLA 4.5 $\mathrm{MeV}$ Photocathode RF gun. This electron source is part of a $20 \mathrm{MeV}$ compact electron linac described before(1). It will be used for studies of the interaction of relativistic beams, plasmas and the generation of coherent radiation. All the components of this system have been built and tested. Full assembly will be completed during the fall of 1993 . Our initial work has been dedicated to a characterization of the photocathode rf gun, which is the electron source for the system. As part of this work we have measured the electron beam emittance and the quantum efficiency of a copper cathode under different conditions. Detailed descriptions of the results of these measurements and the techniques used are reported in other papers presented at this conference. We will limit this

*Work Supported by SDIO/IST through ONR Grant No. N0001490-J-1952 and US DOE Grant DE-FG03-92ER-40493 paper to a summarization of the overall performance of the gun and our plans for its' future development. The photocathode rf gun has also been used to study a thin plasma lens(2). This experiment demonstrated electron beam focusing and confirmed theoretical expectations.

\section{ACCELERATOR SYSTEM DESCRIPTION A. RF System}

The gun is powered by a SLAC XK5 type klystron producing 24 MWatts of rf power with a pulse duration of 4 $\mu s$. The rf system is driven by a signal produced by a master oscillator clock at a frequency of $38.08 \mathrm{MHz}$. This signal is multiplied 75 times to produce the klystron operating frequency of $2.856 \mathrm{GHz}$ and is then sent to a $1 \mathrm{kwatt}$ solid state amplifier. The amplifier signal in turn feeds the klystron. This ensures the timing and a feed back loop stabilizes the laser pulse to $\mathrm{rf}$ jitter to less than $4 \mathrm{ps}$.

\section{B. RF Photo-Injector}

The photocathode if gun is based on the Brookhaven design(3). It consists of a one and a half cell standing wave accelerator producing a beam with an energy up to $4.5 \mathrm{MeV}$. Producing accelerating gradients of up to $100 \mathrm{MV} / \mathrm{m}$ are achieved.

\section{Laser System}

The drive laser is a mode locked Nd-YAG oscillator cavity. To compress the pulse the laser is matched into a $500 \mathrm{~m}$ fiber to produce a frequency chirp. The chirped pulse is then amplified a million times by a regenerative amplifier and 


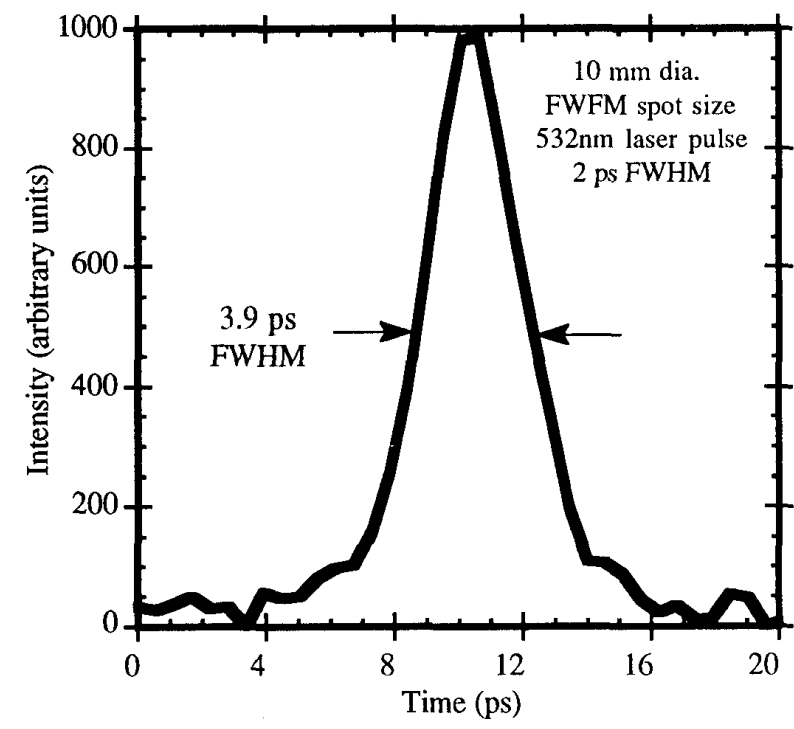

Figure 2.

sent to a grating pair where it is compressed. Once it leaves the compression stage the laser beam is frequency upconverted to green by a $\mathrm{KD}^{* \mathrm{P}}$ crystal. Then it is doubled again to UV, $266 \mathrm{~nm}$, by a second frequency doubling crystal. The up conversion efficiency is typically $10 \%$. The laser was measured with a streak camera and is shown in figure 1 .

\section{Diagnostics}

The electron beam diagnostics are as follows. The main diagnostics are the phosphor screens which monitor the spot size. An Integrating Current Transformer, ICT, is used to measure the electron beam charge along with faraday cups at various locations. Some of the phosphor screens are floated so that they can double as faraday cups. A dipole is used to

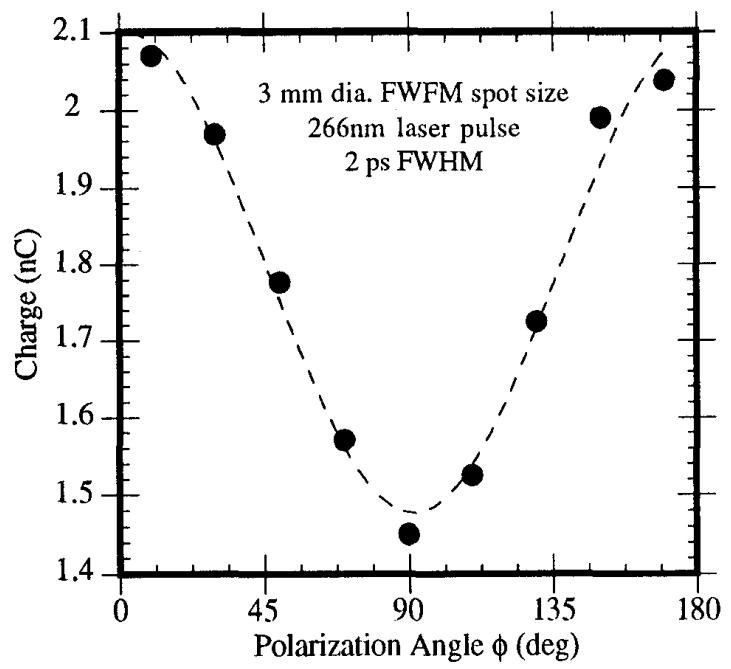

Figure 3. measure the energy and energy spread of the beam.

\section{QUANTUM EFFICIENCY}

\section{A. Quantum Efficiency Results}

The quantum efficiency measurements are described in detail in (4). The quantum efficiency has been measured as a function of input laser energy, illumination angle, and polarization. In the first series of measurements the cathode was initially damaged by over focusing the laser to a small spot size. This produced local melting and damaged the copper cathode surface. The damage limited the possibility of producing a beam with the design spot size of about $3 \mathrm{~mm}$, since most electrons where produced by the damaged area. The resulting effective spot size was about $0.3 \mathrm{~mm}$. This small spot size led to large space charge effects at small charge, so in this series of measurements space charge was always a dominant effect.

\begin{tabular}{|c|c|c|}
\hline $\begin{array}{c}\text { Angle of } \\
\text { Incidence, Degrees }\end{array}$ & Polarization & $\begin{array}{c}\text { Quantum } \\
\text { Efficiency }\end{array}$ \\
\hline \hline 2 & $\mathrm{~S}$ & $9 \times 10^{-5}$ \\
\hline 2 & $\mathrm{P}$ & $9 \times 10^{-5}$ \\
\hline 70 & $\mathrm{~S}$ & $9 \times 10^{-5}$ \\
\hline 70 & $\mathrm{P}$ & $1.3 \times 10^{-4}$ \\
\hline
\end{tabular}

Table 1 .

The main results for the damaged cathode quantum efficiency are given in Table 1 . One can see that the quantum efficiency depends on the incidence angle and the light polarization. The largest quantum efficiency, $10^{-4}$, is obtained for 70 degrees incident angle and $P$ polarized light. The dependence of the quantum efficiency on the polarization angle $\phi$ is shown in Fig. 3 and can be fitted with a $\cos ^{2} \phi$ dependence which implies single photon emission.

\section{ENERGY and ENERGY SPREAD}

\section{Dark Current Peak Momentum Fluctuations}

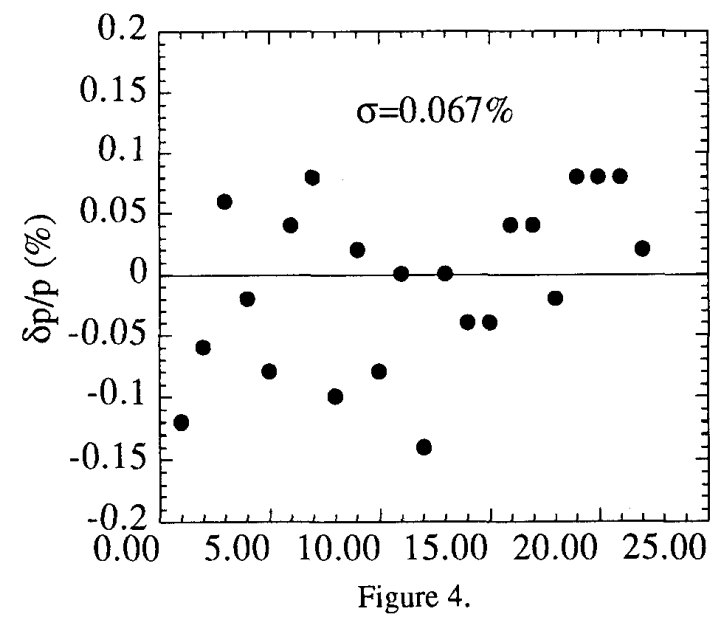


The time jitter of the laser pulse and the fluctuations in the rf voltage are of deep concern for future FEL experiments. In Fig. 4 we show the fluctuations in the dark current energy end point, showing the level of the rf system fluctuations. In Fig. 5 we show the jitter in peak momentum of the photoelectrons, which includes also the laser pulse jitter. One can see that the resulting rms momentum fluctuation is $0.25 \%$ and is due mainly to the residual laser pulse jitter. The dependence of the bunch energy on the initial rf phase is shown elsewhere(5). This dependence shows that the accelerating field in the two cells is unbalanced with a larger field in the full cell. Introducing this unbalance the data can be fitted using the analytical theory of $\mathrm{Kim}$. From the beam energy measurements we can also determine the gun shunt impedance which is evaluated to be $36 \mathrm{M} \Omega / \mathrm{m}$, smaller than the value expected from the Superfish calculations. The beam energy spread measured at low current, $50 \mathrm{pC}$, is less than $0.2 \%$. However, due to the large space charge effects it increases rapidly with charge, when using the damaged cathode.

\section{EMITTANCE}

The emittance has been measured(5) using a pepper pot. Measurements at a charge smaller than $50 \mathrm{pC}$, show an emittance dominated by rf effects with a minimum of 1.5 $\pi \mathrm{mm}$-mrad rms . At larger charge the emittance is dominated by space charge effects, in the case of the damaged cathode and unbalanced gun. These results as well as the results for the energy spread are compatible with the assumption of an initial spot size of $0.3 \mathrm{~mm}$ radius, rms. The rf photoinjector is clearly a very brilliant electron source, capable of producing beams with time structures determined by the incident laser pulse. Metal cathodes are very robust but put strong requirements on the laser fourth harmonic generation efficiency. Importance of reducing amplitude fluctuations and time jitter is also noted. The potoinjector produces a beam of supiorer quality as exemplified by the successful plasma lens

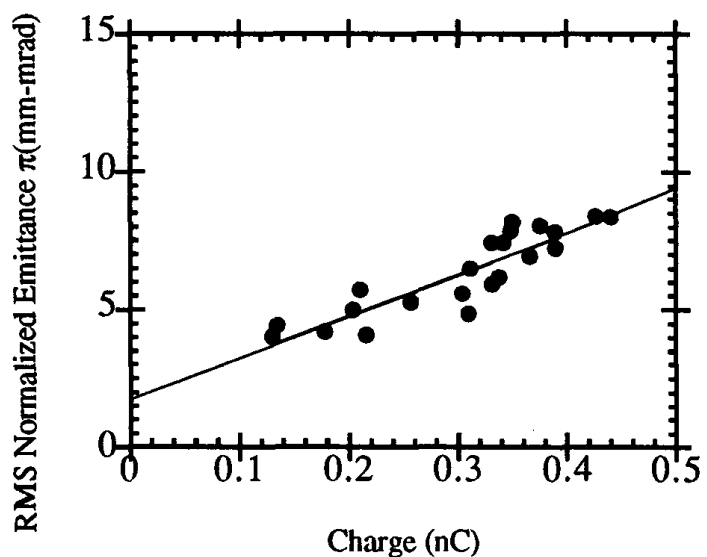

Figure 6.

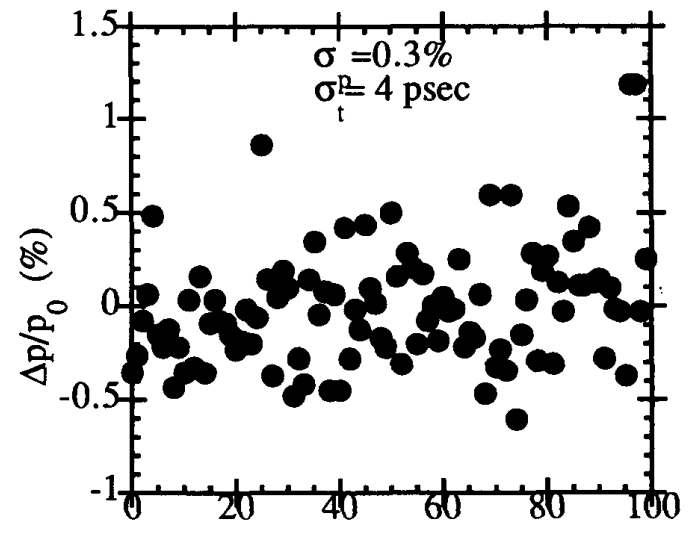

Shot Number (in order of ascending Q)

Figure 5.

experiments completed at UCLA.

\section{CONCLUSION}

The UCLA photo-injector has been operated successfully. The measurements show that the emittance scales as expected. The important thing to note is that for these set of emittance runs the rf photo-injector had a field imbalance. The filled in the full cell was 1.8 times that in the half cell. This contributed to emittance blowup as did the phosphor screens used to measure the emittance. The phosphor screens were placed onto the beamline at a $45^{\circ}$ angle. This created broadening of the line widths

\section{References}

1. S. C. Hartman et al., Photocathode Driven Linac at UCLA for FEL and Plasma Wakefield Acceleration Experiments, Particle Accelerator Conference Sanfransisco, CA., 1991), pp. 2967.

2. G. Hairapetian et al, Experimental Demonstration of Plasma Lens Fucusing, Particle Accelerator Conference Washington, DC., 1993),

3. K. Batchelor et al, European Particle Conference Accelerator Conference Rome, Italy, June 7-12, 1988),

4. P. Davis et al, Quantum Efficiency Measurements of a Copper Photocathode in an rf Electron Gun, Particle Accelerator Conference Washington, DC., 1993),

5. S. C. Hartman et al., Emittance Measurements of the 4.5 MeV UCLA rf Photoinjector, Particle Accelerator Conference Washinton, DC., 1993), 